\title{
Etiology of bloodstream infection and antibiotic susceptibility pattern of the isolates
}

\author{
Prashubha Bhandari', Sarita Manandhar², Basudha Shrestha ${ }^{3}$, Nabeen Dulal ${ }^{4}$ \\ ${ }^{1}$ Student, Department of Microbiology, National College, Tribhuvan University, Khusibu, Kathmandu, '2Lecturer, Department of Microbiology, \\ National College, Tribhuvan University, Khusibu, Kathmandu, ${ }^{3}$ Lecturer, Microbiologist, Kathmandu Model Hospital, Kathmandu, Nepal, ${ }^{4}$ PhD \\ student, RMIT University, Melbourne, Australia
}

Background: Bloodstream infection (BSI) is a significant cause of morbidity and mortality. In Nepal, very few studies on BSIs have restricted the understanding of their cause, prevention and treatment. This cross-sectional study was conducted to isolate BSIs causing pathogens and determine their antibiotic susceptibility pattern in patients visiting Kathmandu Model Hospital during December 2012 to May 2013. Materials and Methods: Standard laboratory procedure was used to screen, isolate and identify the bacteria from 1,205 patients. The antibiotic susceptibility pattern (AST) was analyzed by modified Kirby Bauer technique and data were analyzed using SPSS version-16. Results: Out of 1,205 blood samples, 186 (15.4\%) were culture positive. The most common bacteria isolated were: Salmonella spp., Escherichia coli, Klebsiella pneumoniae and CoNS. Gram-negative bacteria were the predominant causes of BSIs. Salmonella Typhi was isolated in $71 \%$ cases of bloodstream infection followed by Salmonella Paratyphi A in $16 \%$, Escherichia coli in $5.3 \%$ and Klebsiella pneumoniae in $0.5 \%$. The gram-positive organism responsible for causing BSI was coagulase-negative staphylococcus in $7 \%$ cases. There was no significant association between bacteremia and gender of the patients. During ASTs, Gram-negative bacteria were sensitive to Chloramphenicol with only $0.5 \%$ resistivity. Salmonella Typhi $(85.6 \%$ of isolates) showed resistance to Nalidixic acid. Gram-positive bacteria showed $100 \%$ sensitivity towards Chloramphenicol and Gentamicin and were least sensitive to Amoxicillin. Conclusion: Salmonella spp., was major cause of BSIs. Increase in antibiotic resistivity for BSI causing pathogens has necessitated continuous monitoring of the susceptibility of organisms towards antibiotics.

Key words: Bloodstream infections, Escherichia coli, Salmonella Typhi,

Salmonella Paratyphi A, Klebsiella pneumoniae, CoNS

\section{INTRODUCTION}

Bloodstream infection is a potential life-threatening condition with mortality rate ranging from 20 to $50 \%$ and is one of the major causes of death throughout the world. ${ }^{1}$ There are approximately 21.6 million cases of typhoid fever resulting in 200,000 death every year. ${ }^{2}$ In developing countries like Nepal where the annual incidence ranges from 12 to 622 per 100,000, Salmonella Typhi is reported as major cause of bacteremia followed by Salmonella Paratyphi A. ${ }^{3}$

Primary infections, of the respiratory tract, urinary tract and gastro-intestinal tract, often result in bloodstream infections and may cause fever., ${ }^{4,5}$ The toxins produced by bacteria triggers the release of cytotoxin, causing fever, chills, malaise, and lethargy, with difficulty in breathing especially in childern. ${ }^{6}$ Most of the cases of bloodstream infection are caused by Salmonella spp., Escherichia coli, Staphylococcus aureus, Klebsiella pneumoniae. 4,5 Other bacteria responsible for the infection of blood are Citrobacter spp., Pseudomonas spp., coagulase-negative staphylococci. ${ }^{7,8}$

The emergence of resistant bacteria makes it a requisite to know the prevailing antibiotic susceptibility pattern of the pathogens causing bloodstream infection. However, the etiology of bloodstream infections are poorly characterized 
in Nepal, mainly due to limited laboratory resources, poor recording systems and an inadequate number of trained personnel. ${ }^{9}$

This study seeks to investigate the prevalence of BSIs during spring and the monsoon season in patients with various cultural and socio-economic backgrounds. The aim of this study is to investigate the incidence of BSI's, their effect on public health and the effectiveness of antibiotics towards the organisms. This study will contribute in understanding organisms with different antibiotic resistance patterns. The pathogens can be used to determine the genes involved in resistance development and will help identify appropriate treatment of BSIs.

\section{MATERIALS AND METHODS}

The cross-sectional study was conducted in the Microbiology Laboratory of Kathmandu Model Hospital, from December 2012- May 2013. The study included blood samples collected from 1205 patients of different age groups and both genders visiting Hospital, following standard laboratory technique (WHO). Information was collected from patients using a questionnaire: included their medical history and any recent antimicrobial therapy. A blood sample was then inoculated in Brain Heart infusion broth in the ratio $1: 10 .{ }^{10}$ The broth was incubated at $37^{\circ} \mathrm{C}$ for 7 days. Routine subcultures were performed in MacConkey agar and Blood agar (HiMedia, India). Identification of the bacteria was done using different biochemical tests following standard bacteriological procedure. ${ }^{11,12}$ Susceptibility tests of the different isolates were performed by a modified Kirby-Bauer Disk diffusion technique using Mueller Hinton Agar (MHA). The antibiotics (HiMedia, India) were selected and the results were interpreted according to Clinical and laboratory standards Institute (CLSI) 2012 guidelines. ${ }^{13}$ Data analysis was done by using SPSS (Version 16).

\section{RESULTS}

\section{Pathogens distribution}

Of the total 1,205 blood samples processed, 186 (15.4\%) were culture positive. The occurrence of bloodstream infection with respect to gender of the patients was not significantly associated $(\mathrm{p}=0.063)$. The significance threshold was set at 0.05 . However, a higher percentage of males $(17.1 \%)$ was found to be infected in comparison to females(Figure 1). Amongst the total population included $51(4.2 \%)$ were in-patients and 1,154 (95.8\%) were out-patients. There was no significant relation between occurrences of infection in in-patients and out-patients $(\mathrm{p}>0.05)$.
Among the 186 isolates, the most predominant organism was Salmonella Typhi accounting upto $132(71 \%)$ isolates. Similarly $30(16.1 \%)$ isolates of Salmonella Paratyphi A, $10(5.4 \%)$ of Escherichia coli, 13 (7\%) of coagulase-negative staphylococci, and $1(0.5 \%)$ of Klebsiella pneumoniae were also isolated (Table 1).

\section{Antibiotic susceptibility test}

Different antibiotics were used to study the pattern of resistance among the isolates. Chloramphenicol was found to be the most effective drug for both Gram-positive and Gram-negative isolates, especially Salmonella spp., where only $0.57 \%$ of it showed the resistance towards chloramphenicol. Similarly, Gentamicin was effective towards Escherichia coli and Klebsiella pneumoniae with only $9.1 \%$ of these isolates combined together showed the resistance. Gram-negative isolates showed higher percentage of resistance towards Nalidixic acid (86\% of isolates) followed by Ciprofloxacin (27.24 \% of isolates) and Ofloxacin (16.76\% of isolates). However, Amoxicillin was least effective towards Gram-positive with only $23.08 \%$ of total isolates showing the sensitivity.

Chloramphenicol and Gentamicin along with Cefotaxime, Cefixime, Ciprofloxacin and Ofloxacin were effective towards Escherichia coli (Figure 2). Moreover, a single isolate of Escherichia coli was found to be multi drug resistant. It was resistant towards Amoxicillin, Ceftriazone, Cefotaxime, Ciprofloxacin and Ofloxacin.

The Majority of cases of enteric fever caused by Salmonella Typhi were found in outpatients. However, $33.33 \%$ of

\begin{tabular}{lcc}
$\begin{array}{l}\text { Table 1: Distribution of pathogens among the } \\
\text { infected patients }\end{array}$ & \multicolumn{2}{c}{ Infection } \\
\cline { 2 - 3 } Organism isolated & Frequency & Percentage \\
\hline Salmonella Typhi & 132 & 71 \\
Salmonella Paratyphi A & 30 & 16 \\
Escherichia coli & 10 & 5.4 \\
Klebsiella pneumoniae & 1 & 0.5 \\
Coagulase negative Staphylococci & 13 & 7 \\
\hline
\end{tabular}

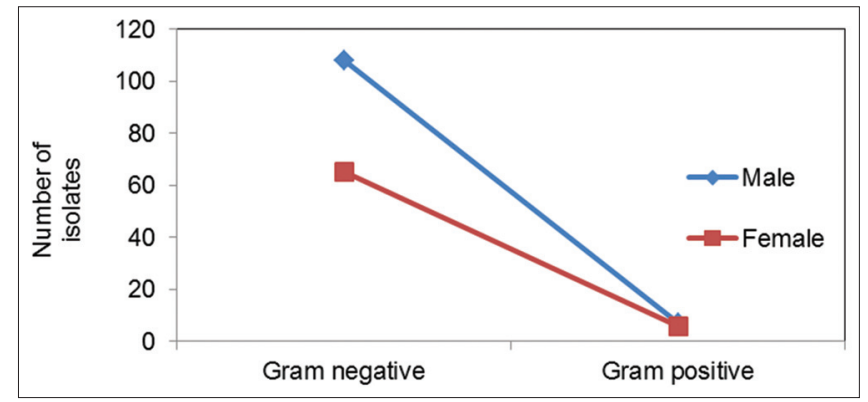

Figure 1: Distribution of pathogens in terms of gender 
Salmonella Typhi isolates in this study were resistant towards Ciprofloxacin, 85.6 \% towards Nalidixic acid and 15.90\% towards Ofloxacin. Among 132 isolates of Salmonella Typhi, $3.03 \%$ showed intermediate results towards Ofloxacin and $0.75 \%$ towards Ciprofloxacin. Chloramphenicol, Amoxicillin, third generation Cephalosporins and Cotri-moxazole were effective towards all the isolates of Salmonella'Typhi (Figure 3).

Unlike Salmonella Typhi, Amoxicillin was one of the least effective drugs for Salmonella Paratyphi A. Ninety percent of isolates of Salmonella Paratyphi A showed resistance towards Nalidixic acid, $13.33 \%$ of isolates towards Amoxicillin followed by $6.66 \%$ towards Ciprofloxacin and Ceftriazone each (Figure 4). Chloramphenicol, Cotrimoxazole, Ofloxacin and Cefixime were highly effective towards Salmonella Paratyphi A.

Amongst the Gram-positive isolates, $23.07 \%$ of Coagulase negative staphylococci isolate showed susceptibility of towards Cephalexin, Cloxacillin and Ciprofloxacin. However, $77 \%$ of the isolates were highly resistant towards Amoxicillin (Figure 5).

A single isolate of Klebsiella pneumoniae was found to be multi drug resistant as it showed resistant towards Amoxicillin, Cefixime, Ceftriazone and Ciprofloxacin. Chloramphenicol, Gentamicin, Cotri-moxazole and Ofloxacin were effective towards the isolate.

\section{DISCUSSION}

Gram negative bacteria were found to be the major cause of bloodstream infection in this study. Salmonella Typhi

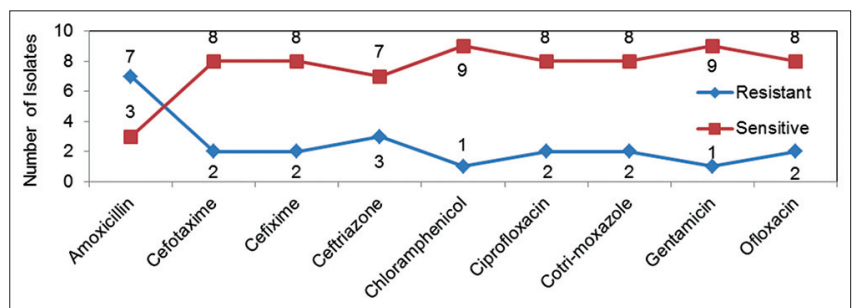

Figure 2: Antibiotic Susceptibility Pattern of E. coli

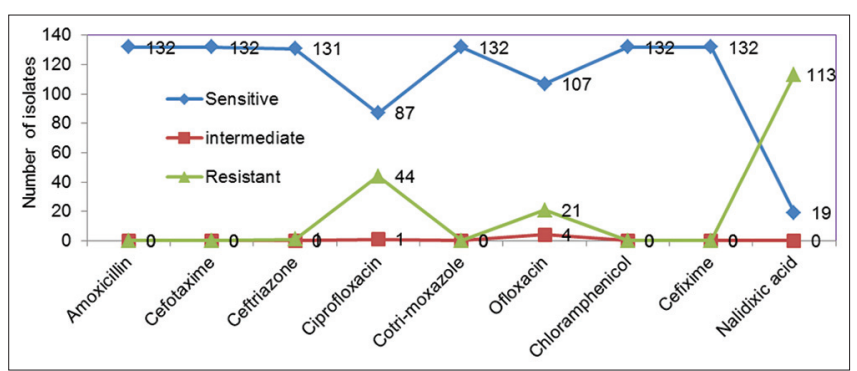

Figure 3: Antibiotic Susceptibility Pattern of Salmonella Typhi is the most common organism isolated which is $71 \%$ $(132 / 186)$ of the total isolates. This result is similar to a study conducted by Amatya et al., in 2005. It found Salmonella Typhi, Salmonella Paratyphi A, Escherichia coli as the pathogens causing BSI's. ${ }^{3}$ Unlike this study where no Gram-positive bacteria were reported, our study reports 13 isolates of CoNS causing BSI. Salmonella spp is the most common isolate indicating that enteric fever is endemic in various parts of Nepal., ${ }^{2,4,5,15}$ All the cases of enteric fever were community acquired. An experiment on 112 cases of typhoid fever in out-patients at Dhulikhel Hospital in 2004 showed a higher percentage of infection in males than in females with a ratio of $3: 1 .^{5}$ This result is similar to the present study as the ratio of infected males to infected females was approximately reported as $2: 1$.

A retrospective study at western region of Nepal from 2010 to 2012 showed Salmonella spp., Klebsiella pneumoniae, Pseudomonas spp., Acinetobacter, Staphylococcus aureus, Coagulase-negative staphyloccous as major cause of bacterimia. Their study showed paratyphoid fever as major cause of bacterimia whereas other study showed typhoid as major infection in patients. ${ }^{15}$

The higher rate of infection by Salmonella spp might have occurred due to unhygienic practices, unavailability of safe drinking water, and consumption of contaminated food particles. ${ }^{5}$ The multi-drug resistant typhoid epidemic in Bharatpur in 2002, affected more than 6,000 patients in period of 4 to 5 weeks and water was reported as a sole source of infection. ${ }^{5}$ Higher occurrence of typhoid in the present study might have also occurred be due to the fact that the study was carried out during monsoon season. During this season where heavy rainfall occurs, there is

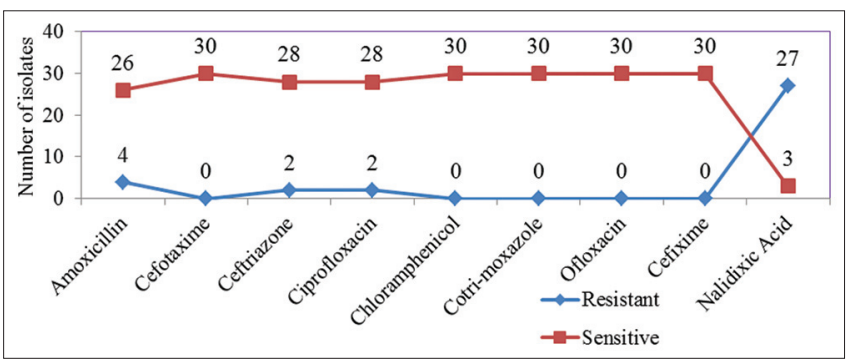

Figure 4: Antibiotic susceptibility pattern of Salmonella Paratyphi A

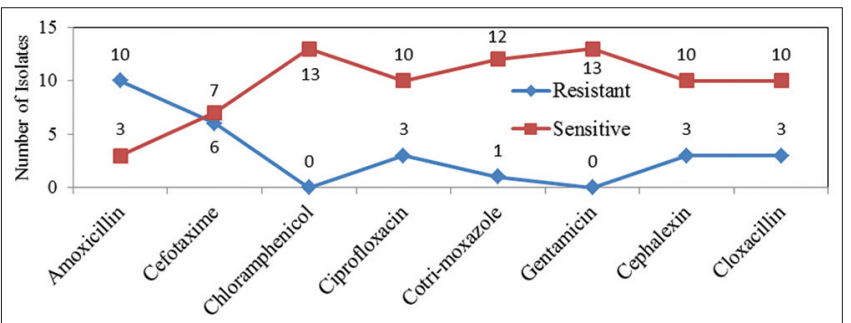

Figure 5: Antibiotic Susceptibility Pattern of CoNS 
a greater chance of contamination of water. Climatic conditions have been shown to have a higher influence in causing typhoid fever in Nepal. ${ }^{15}$

Bacteremia caused by coagulase-negative staphylococci is life threatening if not treated promptly. It is commonly responsible for infection in the immuno-compromised and might cause neonatal sepsis. ${ }^{16}$ This was also confirmed by $53.84 \%$ of CoNS isolates from neonates while, other Staphylococcus spp isolated in the laboratory were considered to be contaminants after studying the leukocytes count of the patients.

Isolated pathogens showed different susceptibility patterns towards antibiotics used. Recently, Chloramphenicol has been used to treat both the gram-positive and gram-negative organisms since, micro-organisms like Salmonella spp., have acquired resistance towards fluoroquinolones. However, Gentamicin still remains effective towards the isolates. Due to prolonged use of the drugs, bacteria acquire resistance towards multiple drugs, causing complications in the treatment of the diseases.

Multi drug resistant bacteria can be defined as those non-susceptible to at least one agent in three or more antimicrobial categories. ${ }^{17}$ In recent years many case studies has shown failure in the treatment of enteric fever with isolates susceptible to fluoroquinolones and resistant to Nalidixic acid. ${ }^{2,18}$ Hence routine testing of resistance to Nalidixic acid can serve as a useful screening test for fluoroquinolones. ${ }^{18}$ The increase in the resistivity of the antibiotics like Ciprofloxacin from $0 \%$ in 2005 to $33.33 \%$ in 2013 might have occurred due to the long-term use of the drugs or the misuse of the drugs by the patients. Ciprofloxacin and Gentamicin can be used as empirical antibiotics for the treatment of BSIs. ${ }^{15}$ But the present study showed increased resistivity of pathogens towards Ciprofloxacin. Whereas, Gentamicin still remains highly effective towards the isolates.

Third generation cephalosporin like Cefexime, Cefotaxime were highly effective in treatment of typhoid and paratyphoid. However, a single isolate of Salmonella Typhi and two of Salmonella Paratyphi A were found to be resistant towards Ceftriazone. Increasing rates of resistance to quinolones and decreased susceptibility to fluoroquinolones, reported in a study ${ }^{18}$ might make us consider Chloramphenicol as an alternative drug for the treatment of resistant strains of Salmonella.

Resistance towards quinolones in Salmonella spp., is conferred by a mutation in the genes encoding DNA gyrase (gyrA or gyrB). Point mutation resultingfrom substitution of amino acid sequence in topoisomerase and plasmid encoded Qnrgenes can also confer resistance to Salmonella spp., ${ }^{3}$ Similarly, reduced outer membrane permeability and increased efflux of the antibiotics due to mutation can also make Salmonella resistant towards antibiotics. ${ }^{3}$ The plasmid-specified $q n r$ determinants provide an unusual mechanism of decreased fluoroquinolones effectivity. ${ }^{19,20}$ In Escherichia coli changes in gyr $A$ gene and $\operatorname{par} C$ gene can cause resistance towards flouroquinoloes. The mutation rate of gyr $A$ gene is higher as compared topar $C$ gene. ${ }^{21}$ The resistance in Escherichia coli, Klebsiella pneumoniae is due to the production of beta lactamase, which hydrolyzesthe $\beta$-lactam ring inactivating the antibiotic. ${ }^{22}$ Antibiotic resistance in Staphylococcus spp., is produced by aminoglycoside modifying enzymes, macrolide methylating enzymes, tetracycline efflux genes, and resistance determinants for trimethoprim sulfamethoxazole. Quinolone resistance in Staphylococcus spp., is mediated primarily by alterations in DNA topoisomerases, and also due to the mutation in staphylococcal nor $A$ gene, which results in the active efflux. ${ }^{23}$ The formation of biofilms by coagulase-negative staphylococci have been known to produce multidrug resistance. $^{24}$

Increasing rates of antimicrobial resistance, changing patterns of antimicrobial usage and the wide application of new medical technologies may change the epidemiology and outcome of bloodstream infection. Increasing antimicrobial resistance among blood pathogens is a matter of great concern when starting empirical antibiotic treatment especially among gram negative bacilli as majority of cases of BSIs are caused by them. It is important to continually review and update the epidemiology and outcome of bloodstream infection., ${ }^{4,14}$ Therefore regular monitoring and update of resistance pattern of pathogens is of great importance in the management of BSIs. However, studies with molecular analysis are necessary to further evaluate this finding.

One of the limitations of the current study was to rely on a single blood culture result.

\section{CONCLUSION}

Gram-negative bacteria i.e., Salmonella Typhi was the major organism (71\%) causing BSI. Salmonella Paratyphi A, Escherichia coli, coagulase-negative Staphylococci, Klebsiella pneumonia were the other common etiological agent. As the resistant rate of an antibiotic for bloodstream pathogens is increasing, continuous monitoring of the susceptibility of the organism towards antibiotics has become mandatory, in order to avoid the inappropriate use of the antibiotics. This study however is localized and may not be generalized to all parts of Nepal. 


\section{ACKNOWLEDGEMENT}

We are very grateful towards Kathmandu Model Hospital for providing us with this opportunity. We highly acknowledge staffs of Kathmandu Model Hospital and National College (Department of Microbiology) for their support.

\section{REFERENCES}

1. Del Rio A, Cervera C, Moreno A, Moreillon P and Miró JM. Patients at risk of complications of Staphylococcus aureus bloodstream infection. Clinical Infectious Diseases 2009;489(4):S246-S253.

2. Acharya D, Trakulsomboon S, Madhup SK and Korbsrisate S. Antibiotic susceptibility pattern and the indicator of decreased ciprofloxacin susceptibility of Salmonella enterica serovar Typhi isolated from Dhulikhel Hospital, Nepal. Japanese Journal of Infectious Diseases 2012;65:264-267.

3. Amatya NM, Shrestha B and Lekhak B. Etiological agents of bacteraemia and antibiotic susceptibility pattern in Kathmandu Model Hospital. Journal of Nepalese Medical Association 2007;46(167): 112-118.

4. Pandey S, Raza S and Bhatta CP. The aetiology of the Bloodstream Infections in the Patients Who Presented to a Tertiary Care Teaching Hospital in Kathmandu, Nepal. Journal of Clinical and Diagnostic Research 2013;7(4): 638-641.

5. Sharma N, Koju R, Karmacharya B, Tamang MD, Makaju R, Nepali N, et al. Typhoid fever in Dhulikhel hospital, Nepal. Kathmandu University Medical Journal (KUMJ)2004; 2:188-192.

6. Ayobola ED, Sochi O and Ovuokeroye O. Study of prevalence and antimicrobial susceptibility of blood culture bacterial isolates. Malaysian Journal of Microbiology 2011;7(2): 78-82.

7. Sligl W, Taylor $G$ and Brindley PG. Five years of nosocomial Gram-negative bacteremia in a general intensive care unit: epidemiology, antimicrobial susceptibility patterns, and outcomes. International Journal of Infectious Diseases 2006;10: 320-325.

8. Diekema DJ, Beekmann SE, Chapin KC, Morel K. a, Munson E, Doern GV. et al. Epidemiology and outcome of nosocomial and community-onset bloodstream infection. Journal of Clinical. Microbiology 2003.41(8): 3655-3660.

9. Murdoch DR, Woods CW, Zimmerman MD, Dull PM, Belbase RH, Keenan AJ. et al. The etiology of febrile illness in adults presenting to Patan Hospital in Kathmandu, Nepal. American. Journal of Tropical Medicine and Hygiene 2004;70(6): 670-675.

10. Forbes BA, Sahm D and Weissfeld A. Bailey and Scott's Diagonistic Microbiology 2002; Mosby Inc, USA.

11. Cheesebrough M. Medical Laboratory Manual for Tropical
Countries Microbiology.Cambridge University Press, 1984.

12. Cheesbrough M. District Laboratory Practice in Tropical Countries.Cambridge University Press, 2000.

13. CLSI. Performance standards for antimicrobial susceptibility testing' twenty-second informational supplement. 2012; Vol. 32 CLSI Document M100-S23.

14. Karki S, Rai GK and Manandhar R. Bacteriological analysis and antibiotic sensitivity pattern of blood culture isolates in Kanti children hospital. Journal of Nepal Paediatric Society 2010;30(2): 94-97.

15. Bhatta D, Gaur A and Supram HS. Bacteriological profile of Blood Stream Infection Among febrile patients attending a tertiary care centre of Western Nepal. Asian Journal of Medical Sciences 2013;4(3): 92-98.

16. Björkqvist $M$, Liljedahl $M$, Zimmermann $J$, Schollin $J$ and Söderquist B. Colonization pattern of coagulase-negative staphylococci in preterm neonates and the relation to bacteremia. European Journal of Clinical Microbiology and Infectious Diseases 2010;29(9): 1085-1093.

17. Magiorakos PA, Srinivasan A, Carey RB, Carmeli Y, Falagas ME, Giske CG. et al. Multidrug-resistant, extensively drug-resistant and pandrug-resistant bacteria: An international expert proposal for interim standard definitions for acquired resistance. Clinical Microbiology Infection 2012;18: 268-28.

18. Butt T, Ahmad RN, Mahmood A and Zaidi S. Ciprofloxacin Treatment Failure in Typhoid Fever Case, Pakistan. Emerging Infectious Diseases2003; 9(12): 1621-1622.

19. Munson EL, Diekema DJ, Beekmann SE, Chapin KC and Doern GV. Detection and treatment of bloodstream infection: Laboratory reporting and antimicrobial management. Journal of Clinical Microbiology 2003;41: 495-497.

20. Maskey AP, Day JN, Phung QT, Thwaites GE, Campbell JI, Zimmerman $M$. et al. Salmonella enterica serovar Paratyphi A and S. enterica serovar Typhi cause indistinguishable clinical syndromes in Kathmandu, Nepal. Clinical Infectious Disease 2006;42: 1247-1253.

21. Martinez $\mathrm{JL}$ and Baquero F. Mutation Frequencies and Antibiotic Resistance. Antimicrobial Agents and Chemotherapy 2000;44(7):1771-1777.

22. Yan JJ, Ko WC, Wu JJ, Tsai SH and Chuang CL. Epidemiological investigation of bloodstream infections by extended spectrum cephalosporin-resistant Escherichia coli in a Taiwanese teaching hospital. Journal of Clinical Microbiology 2004;.42(7): 3329-3332.

23. Rouveix B. Clinical implications of multiple drug resistance efflux pumps of pathogenic bacteria. Journal of Antimicrobial agents and Chemotherapy 2007;59: 1208-1209.

24. Kang $\mathrm{Cl}$ and Song $\mathrm{JH}$. Antimicrobial resistance in Asia: Current epidemiology and clinical implications. Infection and Chemotherapy 2013; 45(1): 22-31. 